\title{
Constructing Integrable Lindblad Superoperators
}

\author{
Marius de Leeuw and Chiara Palettei \\ School of Mathematics \& Hamilton Mathematics Institute \\ Trinity College Dublin \\ Dublin, Ireland \\ Balázs Pozsgayl \\ Department of Theoretical Physics 8 \\ MTA-ELTE "Momentum" Integrable Quantum Dynamics Research Group \\ Eötvös Loránd University \\ Budapest, Hungary
}

\begin{abstract}
We develop a new method for the construction of one-dimensional integrable Lindblad systems, which describe quantum many body models in contact with a Markovian environment. We find several new models with interesting features, such as annihilation-diffusion processes, a mixture of coherent and classical particle propagation, and a rectified steady state current. We also find new ways to represent known classical integrable stochastic equations by integrable Lindblad operators. Our method can be extended to various other situations and it establishes a structured approach to the study of solvable open quantum systems.
\end{abstract}

Introduction.- One-dimensional quantum integrable models display exotic physical behaviour. They possess a large number of conserved charges which constrain the dynamical processes. As a consequence, isolated integrable models equilibrate to the Generalized Gibbs Ensemble (GGE) [1 3 ; , and their large scale transport properties can be described by Generalized Hydrodynamics (GHD) 细, 5]. These special properties have been investigated in a number of experiments with cold atoms, see for example [6 9]. However, in experiments there are always integrability breaking interactions within the system, and also between the system and its environment. Recently a number of works were devoted to the question whether the integrability breaking effects can be handled within the GGE and the GHD, see for example 10 17.

The interactions with the environment can be described by the Lindblad equation if the response of the environment is Markovian [18 20]. While most Lindbladians explicitly break integrability, there are cases when the Lindblad equation itself is integrable, or it shows certain features of integrability. Finding solvable examples is important because then the non-equilibrium steady states (NESS) and the relaxation towards them can be studied with exact methods. Exact solutions could be used to justify the approximations involving time dependent GGE or GHD 10, 16, 17, 21, and they are also relevant for quantum circuits 22|. For potential experimental applications see [21, 23]26].

Lindblad systems showing various types of solvability include a) models solvable by free fermion techniques 27 30 b) boundary driven spin chains that allow for the

\footnotetext{
mdeleeuw@maths.tcd.ie

$\uparrow$ balettac@maths.tcd.ie

pozsgay.balazs@gmail.com
}

construction of their NESS [31 36], c) triangular Lindblad superoperators that allow for the computation of the Liouvillian spectrum [37, 38], d) models where the integrability is established separately for different subspaces of the full operator space [39]; and e) Yang-Baxter integrable Lindblad systems with local jump operators in the bulk 40 42. The latter type of models are related to solutions of the Yang-Baxter equation, which is a central algebraic relation in the theory of integrable models 43. So far there has been no structured approach to Yang-Baxter integrable Lindblad systems: the examples in the literature were found through relations to known spin ladder systems 404 , but there was no method for actually constructing new integrable cases.

In the present paper we fill this gap and initiate a systematic classification of Yang-Baxter integrable Lindblad systems. We explore certain sections of the parameter space for such models and we find several new cases. Our main method is borrowed from 44 47, where solutions of the Yang-Baxter equation were classified in various other circumstances. The new Lindblad systems correspond to novel solutions of the Yang-Baxter equation and exhibit interesting features, such as a tunable coupling to the environment and a mixture of coherent and classical transport.

The Lindblad equation.- We consider a spin- $1 / 2$ chain of length $L$, with a Hamiltonian $H=\sum_{j=1}^{L} h_{j, j+1}$, where $h_{j, j+1}$ is the Hamiltonian density acting on the neighboring sites $j$ and $j+1$. We assume periodic boundary conditions. We also assume that our model is in contact with a thermal environment that is Markovian, so that its internal dynamics evolves much faster than that of our model. In this case, the time dependence of the density matrix of our model is well approximated by the 
Lindblad master equation 18, 19, 48, 49:

$$
\dot{\rho} \equiv \mathcal{L} \rho=i[\rho, H]+\sum_{a}\left[\ell_{a} \rho \ell_{a}^{\dagger}-\frac{1}{2}\left\{\ell_{a}^{\dagger} \ell_{a}, \rho\right\}\right] .
$$

Here $\mathcal{L}$ is the so-called Lindblad superoperator. In (1) the commutator term describes coherent time evolution dictated by the Hamiltonian, and the $\ell_{a}$ are the so-called Lindblad or jump operators, which describe dissipative processes mediated by the environment. The summation above runs over all interactions with the environment; we focus on local processes in the bulk with one family of local jump operators $\ell_{a} \equiv \ell_{j, j+1}$, which act on sites $j$ and $j+1$ of the spin-chain.

Classical flows.- Before discussing the integrable cases we explain an important connection to integrable classical stochastic equations, which have a rich history themselves [50|. It turns out that the Lindblad equation is capable of realizing the classical flows on the diagonal of the density matrix. The diagonal elements of $\rho$ can be understood as classical probabilities of finding the system in the given state, and in some models the operator space spanned by the diagonal elements is kept invariant by the Lindblad superoperator. In such cases the flow equation (11) for $\rho$ can be projected to the diagonal elements $\left\langle n_{1}, \ldots, n_{L}|\rho| n_{1}, \ldots, n_{L}\right\rangle \equiv P\left(n_{1}, \ldots, n_{L}\right)$, where $\left|n_{1}, n_{2}, \ldots, n_{L}\right\rangle, n_{j}=\uparrow, \downarrow$ are the vectors of the computational basis. Defining the vector

$$
|P\rangle=P\left(n_{1}, \ldots, n_{L}\right)\left|n_{1}, \ldots, n_{L}\right\rangle,
$$

we obtain a flow equation $\partial_{t}|P\rangle=W|P\rangle$, where $W=$ $\sum_{j=1}^{L} w_{j, j+1}$ is the generator of the classical flow, with its matrix elements given by the corresponding projection of $\mathcal{L}$. Lindblad equations that keep the diagonal of $\rho$ invariant will be called diagonal preserving.

If the initial $\rho$ is diagonal in such a model, then it will stay diagonal and the classical flow will be realized. However, preservation of the diagonal does not imply that the orthogonal complement of the diagonal subspace is also conserved, so for generic configurations we can still expect quantum effects in the time evolution.

An example for a diagonal conserving model was discussed in [51] (see also [39, 52]). Here $h_{j, j+1}=0$ and the model has two families of jump operators

$$
\ell_{j, j+1}^{R}=\sqrt{\varphi_{R}} \sigma_{j}^{-} \sigma_{j+1}^{+}, \quad \ell_{j, j+1}^{L}=\sqrt{\varphi_{L}} \sigma_{j}^{+} \sigma_{j+1}^{-},
$$

with $\varphi_{L, R} \geq 0$. The resulting classical flow was found to be the Asymmetric Simple Exclusion Process (ASEP), with the generator being

$$
\begin{aligned}
w_{j, j+1}=\varphi_{R}[ & \left.\sigma_{j}^{-} \sigma_{j+1}^{+}-n_{j}\left(1-n_{j+1}\right)\right]+ \\
& +\varphi_{L}\left[\sigma_{j}^{+} \sigma_{j+1}^{-}-\left(1-n_{j}\right) n_{j+1}\right] .
\end{aligned}
$$

It is known that the ASEP is related to the Heisenberg XXZ spin chain 53 and thus it is integrable. However, the integrability properties of the superoperator itself were not investigated in [39, 51]. We found that the Lindblad system given by (3) is not Yang-Baxter integrable, but we found integrable "parent" Lindbladians in some special cases, see below.

Lindblad equation and spin ladders. - We treat the Lindblad superoperator as a (non-Hermitian) Hamiltonian of a spin ladder, where the two legs correspond to the "bra" and "ket" sides of the density matrix [40]. We use the computational basis to identify the Hilbert space $\mathcal{H}$ with its dual $\mathcal{H}^{*}$. Then the density matrix $\rho \in \mathcal{H} \otimes \mathcal{H}^{*}$ can be represented by an element of the tensor product $\mathcal{H}^{(1)} \otimes \mathcal{H}^{(2)}$ which in turn is interpreted as the Hilbert space of a spin ladder.

As mentioned, we consider one family of jump operators in the bulk. Then the superoperator can be written in the spin ladder representation as $\mathcal{L}=\sum_{j} \mathcal{L}_{j, j+1}$, with

$$
\begin{aligned}
\mathcal{L}_{j, j+1}= & -i h_{j, j+1}^{(1)}+i h_{j, j+1}^{(2) *}+\ell_{j, j+1}^{(1)} \ell_{j, j+1}^{(2) *} \\
& -\frac{1}{2} \ell_{j, j+1}^{(1) \dagger} \ell_{j, j+1}^{(1)}-\frac{1}{2} \ell_{j, j+1}^{(2) T} \ell_{j, j+1}^{(2) *} .
\end{aligned}
$$

Above the superscript ${ }^{T}$ denotes transpose and the asterisk stands for complex conjugation component wise. For any operator $A$ the notation $A^{(1)}$ or $A^{(2)}$ means that it acts only on $\mathcal{H}^{(1)}$ or $\mathcal{H}^{(2)}$. It is our goal to find integrable spin ladders with a (non-Hermitian) Hamiltonian having the structure (5).

Yang-Baxter integrability.- A large class of quantum integrable models can be constructed from solutions of the celebrated Yang-Baxter equation (YBE) 443. The YBE concerns the so-called $R$-matrix $R(u, v)$, which acts on $V \otimes V \simeq \mathbb{C}^{d} \otimes \mathbb{C}^{d}$, and $u$ and $v$ are the so-called spectral parameters. The YBE reads

$$
\begin{aligned}
& R_{12}\left(u_{1}, u_{2}\right) R_{13}\left(u_{1}, u_{3}\right) R_{23}\left(u_{2}, u_{3}\right)= \\
& =R_{23}\left(u_{2}, u_{3}\right) R_{13}\left(u_{1}, u_{3}\right) R_{12}\left(u_{1}, u_{2}\right)
\end{aligned}
$$

which is an equality for operators acting on $V \otimes V \otimes$ $V$, and each spectral parameter is associated to a space $V$. The solutions generate integrable spin chains; for the details of the construction we refer to [43, 44], and here we just review a few key statements.

If the regularity condition $R(u, u)=P$ holds with $P$ being the permutation operator, then we can define a family of Hamiltonian densities as

$$
h(u)=\left.P \partial_{v} R(v, u)\right|_{v=u} .
$$

Each $h(u)$ describes a nearest neighbor interacting integrable chain with local spaces $\simeq \mathbb{C}^{d}$. The models are generally different for different values of $u$. However, if the $R$-matrix is of difference form $R(v, u)=R(v-u)$, then the $u$-dependence drops out.

The models possess a commuting set of charges $Q_{\alpha}$ (with range $\alpha$ ), such that the Hamiltonian is a member 
of the series. Identifying $Q_{2}=H$, the higher charges can be obtained either from the transfer matrix 443, or they can be generated with the so-called boost operator $\mathbb{B}$ [54 56] as

$$
Q_{\alpha+1}(u) \sim\left[\mathbb{B}, Q_{\alpha}(u)\right], \quad \alpha>1 .
$$

The boost operator is a differential operator and it depends on the Hamiltonian density:

$$
\mathbb{B}:=\partial_{u}+\sum_{j=-\infty}^{\infty} j h_{j, j+1}(u) .
$$

This formal infinite sum gives a well defined commutator in (8).

Construction of new models. - Let us review the method of 4447 to find new integrable Hamiltonians; the extension to Lindblad systems is discussed afterwards. The key idea is to start with a Hamiltonian density $h_{j, j+1}(u)$, then find the first non-trivial higher charge $Q_{3}(u)$ using (8) and (9), and to impose the condition $\left[Q_{2}(u), Q_{3}(u)\right]=0$. This leads to a set of coupled first order, non-linear, differential equations for the components of $h(u)$, which can be solved if an initial Ansatz for $h(u)$ is given. A priori it is not clear whether the commutativity between $Q_{2}(u)$ and $Q_{3}(u)$ is enough to ensure the existence of the remaining higher charges, but in all previous cases this was found to be sufficient. The $R$-matrix is afterwards found by solving the so-called Sutherland-equation, taking into account (7) and the regularity condition. This strategy has proven to be successful in several cases and led to many new solutions of the Yang-Baxter equation [44 47].

Integrable Lindblad superoperators. - For Lindblad systems the key is to find a (non-Hermitian) Hamiltonian density for a spin ladder, which takes the special Lindblad form (5). However, now we also allow for an $u$ dependence of the matrices $h$ and $\ell$, where $u$ is the spectral parameter used in the $R$-matrix. Different values of $u$ will generally give different physical systems, except if the corresponding $R$-matrix is of difference form. We substitute this Ansatz into our integrability constraint and then solve the corresponding set of differential equations for the components of the operators $h(u)$ and $\ell(u)$.

Strictly speaking we are imposing too strong a condition: we require that we find a Lindblad superoperator for any value of $u$, whereas it would be enough to find a solution of the Yang-Baxter equation such that the decomposition (5) works for some value of $u$. However, this strong requirement leads to a structured approach and it allows for a number of non-trivial Lindblad systems.

Once a model is found, we can apply simple transformations which lead to seemingly different matrices, nevertheless describing the same physical behaviour. These transformations include a) unitary basis transformations, b) space- and spin-reflection, c) trivial shifts $h \mapsto h+r$ with $r \in \mathbb{R}, d$ ) telescopic operator shifts $h_{j, j+1} \mapsto$ $h_{j, j+1}+o_{j}-o_{j+1}$, where $o_{j}$ is a one-site operator, eventually adding up to zero on the periodic chain, e) combined shifts

$$
(h, \ell) \mapsto\left(h+i\left(z \ell^{\dagger}-z^{*} \ell\right), \ell+2 z\right), \quad z \in \mathbb{C},
$$

which leave the superoperator invariant, and f) a combined re-scaling

$$
(h, \ell) \mapsto\left(r h, \sqrt{r} e^{i \mu} \ell\right), \quad r \in \mathbb{R}^{+}, \mu \in \mathbb{R},
$$

which only affects the choice of the unit of time. Below we always choose a representation such that the matrices of either $h$ or $\ell$ are as simple as possible.

Partial classification.- We carve out a section of the space of integrable superoperators by restricting to specific forms of $h$ and $\ell$. The two choices we investigate are the following: $A$ ) Lower triangular $\ell$ operators with at most two elements below the diagonal. $B$ ) $h$ and $\ell$ operators that both conserve the total $S^{z}$ quantum number.

Curiously both restrictions $A$ ) and $B$ ) only allow for very specific Hamiltonian densities: We found that either $h$ is diagonal, or it is given by

$$
h_{j, j+1}=\frac{1}{2}\left[e^{i \phi} \sigma_{j}^{+} \sigma_{j+1}^{-}+e^{-i \phi} \sigma_{j}^{-} \sigma_{j+1}^{+}\right], \quad \phi \in \mathbb{R} .
$$

This Hamiltonian density describes a free fermionic hopping model; the factor of $1 / 2$ is added only for later convenience. The angle $\phi$ can be understood as a homogeneous twist along the chain, and the $\phi=0$ point corresponds to the XX spin chain. Alternatively, the model can be interpreted as the XX chain perturbed by a Dzyaloshinskii-Moriya interaction term, [57|.

We use the particle picture to interpret our models. We choose the vacuum state as the state with all spins down, and an up spin is interpreted as a particle; the operators $n_{j}=\left(1+\sigma_{j}^{z}\right) / 2$ measure the local occupation numbers.

We now give a list of integrable Lindbladians, almost all of which are new |58|. The $R$-matrices of the models are presented for all cases in 59 .

Model A1.- If we require that $\ell$ is lower triangular with just one element below the diagonal then we find only one solution. It can be represented with $h$ given by (12) and the Lindblad operator being

$$
\ell_{j, j+1}=\sigma_{j}^{-} \sigma_{j+1}^{+}-i e^{i \phi}\left(1-n_{j}\right) n_{j+1} .
$$

The corresponding $R$-matrix is of difference form and seems to be new. Direct computation shows that the model is diagonal preserving, and for the diagonal flow we find the generator

$$
w_{j, j+1}^{\mathrm{TASEP}}=\sigma_{j}^{-} \sigma_{j+1}^{+}-n_{j}\left(1-n_{j+1}\right) .
$$

This is the generator of the Totally Asymmetric Simple Exclusion Process (TASEP) |50|. To our best knowledge 
this is the first realization of the TASEP using an integrable Lindbladian.

Model A2.- If we require a lower triangular $\ell$ with two elements below the diagonal and a non-diagonal $h$ we again find only one solution, which has an $R$-matrix of difference form. The Hamiltonian density is given by (12) with $\phi=\tau \pi / 2$ and the Lindblad operator is now

$$
\ell_{j, j+1}=n_{j+1}+\tau \sigma_{j}^{-} \sigma_{j+1}^{+}+\sigma_{j}^{-} \sigma_{j+1}^{-},
$$

where $\tau= \pm 1$. This jump operator describes particle propagation to the right and local two-body loss. The model is diagonal preserving with the generator being

$$
w_{j, j+1}=-n_{j}+\sigma_{j}^{-} \sigma_{j+1}^{+}+\sigma_{j}^{-} \sigma_{j+1}^{-} .
$$

This corresponds to the totally asymmetric limit of the diffusion-annihilation model treated in 6062. To our best knowledge this is the first time that this generator is embedded into an integrable Lindbladian.

Let us now turn to the models where both $h$ and $\ell$ conserve the global $S^{z}$ operator.

Model B1.- In this case $h=0$ and the Lindblad operator is

$$
\ell=\left(\begin{array}{llll}
\tau & 0 & 0 & 0 \\
0 & 0 & 1 & 0 \\
0 & 1 & 0 & 0 \\
0 & 0 & 0 & \kappa
\end{array}\right)
$$

where $\tau= \pm 1, \kappa= \pm 1$. In the case of $\tau=\kappa=1$ the superoperator is equivalent to the Hamiltonian of the $S U(4)$-invariant chain; this case was listed in [42]. The $R$-matrix is of difference form for all four choices, and for $\tau \kappa \neq 1$ it seems to be new. The model is diagonal preserving in all four cases and the generator is

$$
w_{j, j+1}=\sigma_{j}^{+} \sigma_{j+1}^{-}+\sigma_{j}^{-} \sigma_{j+1}^{+}-n_{j}-n_{j+1}+2 n_{j} n_{j+1} .
$$

This is the generator of the Symmetric Simple Exclusion Process (SSEP) |50|. To our best knowledge it is the first time that the SSEP is realized by an integrable Lindbladian. The parameters $\tau, \kappa$ have an effect on the off-diagonal sectors of the superoperator, and they influence the Liouvillian spectrum.

Model B2.- The Hamiltonian is given by (12) and the Lindblad operator is given by

$$
\frac{\ell(u)}{\beta(u)}=\left(\begin{array}{cccc}
\operatorname{ch}(u) & 0 & 0 & 0 \\
0 & 1 & i \operatorname{sh}(u) e^{i \phi} & 0 \\
0 & -i \operatorname{sh}(u) e^{-i \phi} & -1 & 0 \\
0 & 0 & 0 & -\operatorname{ch}(u)
\end{array}\right)
$$

with $\beta(u)=(\gamma /(2 \gamma \operatorname{ch}(2 u)+2))^{1 / 4}$ and $\gamma \geq 0$ being a fixed coupling constant. For $u=\phi=0$ this system is equivalent to the $\mathrm{XX}$ model with dephasing noise treated in [40], which in turn corresponds to the Hubbard model with imaginary coupling. The $R$-matrix is known and it is not of difference form 63].
For $u \neq 0$ the model can be interpreted as the inhomogeneous version of the Hubbard model [42, 64, and it involves a mixture of coherent and stochastic particle propagation. The particle current $J_{k}$ can be found from the continuity relation $d n_{k} / d t=J_{k-1}-J_{k}$ and the Lindblad equation, and it is given by

$$
J_{k}=\left(1-2 \beta^{2}(u) \operatorname{sh}(u)\right) J_{k}^{0}+\beta^{2}(u) \operatorname{sh}^{2}(u)\left(n_{k}-n_{k+1}\right) .
$$

Here

$$
J_{k}^{0}=\frac{i}{2}\left(e^{i \phi} \sigma_{k}^{+} \sigma_{k+1}^{-}-e^{-i \phi} \sigma_{k}^{-} \sigma_{k+1}^{+}\right),
$$

is the current of the coherent time evolution dictated by (12), and the remaining terms describe stochastic transport.

Model B3.- This is a completely new model. The Hamiltonian is given again by (12) and

$$
\ell=\sqrt{\frac{\gamma}{2}}\left(\begin{array}{cccc}
\gamma & 0 & 0 & 0 \\
0 & 1 & i(\gamma-1) e^{i \phi} & 0 \\
0 & -i(\gamma+1) e^{-i \phi} & -1 & 0 \\
0 & 0 & 0 & \gamma
\end{array}\right)
$$

Here $\gamma \geq 0$ is a coupling constant. The $R$-matrix is of difference form and it appears to be new. At the special point $\gamma=1$ the model is diagonal preserving and it describes the TASEP. For generic values of $\gamma$ the model describes a mixture a quantum and classical transport. The particle current is now given by

$$
\begin{gathered}
J_{k}=\left(1-\gamma^{2}\right) J_{k}^{0}+\frac{\gamma(1+\gamma)^{2}}{2} n_{k}\left(1-n_{k+1}\right)- \\
-\frac{\gamma(1-\gamma)^{2}}{2}\left(1-n_{k}\right) n_{k+1}
\end{gathered}
$$

with $J_{k}^{0}$ given by (21). The stochastic terms explicitly break space reflection symmetry: they describe the current in the ASEP.

Steady states. - The dynamics of the models lead to the formation of non-equilibrium steady-states $\rho$, which satisfy $\mathcal{L} \rho=0$. Here we focus on Models B2 and B3.

In Model B2 the NESS is the infinite temperature state [40|. In contrast, in Model B3 we find that the NESS is a current carrying mixed state, which actually degenerates into a pure state if the compatibility condition $e^{i(\phi+\pi / 2) L}=1$ holds. For the XX model $(\phi=0)$ this means $L \equiv 0 \bmod 4$. In such a case let us consider $\rho_{h}=|\Psi\rangle\langle\Psi|$ where $|\Psi\rangle$ is a spin helix state given by

$$
|\Psi\rangle=\otimes_{j=1}^{L} \frac{1}{\sqrt{2}}\left(\begin{array}{c}
1 \\
e^{i j(\phi+\pi / 2)}
\end{array}\right) .
$$

Direct computation shows that $H|\Psi\rangle=0$ and $\mathcal{L} \rho_{h}=0$ for every $\gamma$. The superoperator conserves particle number, thus the NESS' in the sectors with fixed spin are given by the appropriate projections of $\rho_{h}$. Numerical 
studies on small systems show that these are the unique steady states in the various spin sectors.

The particle current in the projected states can be computed easily, and in the thermodynamic limit we find

We conjecture that this formula holds generally, even if the compatibility condition is not met.

We observe a remarkable phenomenon: there is a finite particle current even if the Lindblad coupling $\gamma$ is tuned back to zero. This phenomenon is understood as a pumpring effect for, fof. The jump operators are couplect to the coneremt current, thus they ibuitu up its mean value over time. The current itself is conserved, thus it cannot decay. Eventually a current carrying state is produced no matter how small the coupling is. Our model presents an eactly solvable case for this phenomenon earlier discussed in 65 6.6. For the same effect with bowndary driving see [36.

Discussion.- We presented a method for the construction of integrable Lindblad systems, and found new models for spin- $1 / 2$ chains. As a by-product we also discovered apparently new solutions of the Yang-Baxter equation which can also describe Hermitian spin ladders for different choices of their parameters.

In some cases our integrable Eindblat superoperators

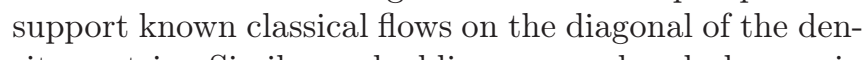
sity matrix. Similar embeddings were already known in the literature 39, 51, 52, but the existence of YangBaxter integrable superoperators behind the classical flows is a new result.

It would be important to continue the classification commenced in this work; so far we only explored a limited parameter space for the models. Our methods could potentially lead to further integrable Lindblad systems, including models with particle creation and annihilation processes, multiple families of jump operators, higher dimensional local spaces, and models with boundaries. Furthermore, it is desirable to find the Bethe Ansatz solution to Model B3, which would lead to an understanding of its relaxation dynamics.

Acknowledgments. We would like to thank Zala Lenarčič, Andrea Nava, Lorenzo Piroli, Tomaž Prosen, Florentin Reiter, Ana L. Retore and Marco Rossi for useful discussions. M.dL. was supported by SFI, the Royal Society and the EPSRC for funding under grants UF160578, RGF $\backslash \mathrm{EA} \backslash 181011, \mathrm{RGF} \backslash \mathrm{EA} \backslash 180167$ and 18/EPSRC/3590. C.P. is supported by the grant RGF

[1] M. Rigol, V. Dunjko, V. Yurovsky, and M. Olshanii, "Relaxation in a completely integrable many-body quantum system: An ab initio study of the dynamics of the highly excited states of 1D lattice hard-core bosons," Phys. Rev. Lett. 98 (2007) no. 5, 050405. arXiv: cond-mat/0604476

[2] E. Ilievski, J. De Nardis, B. Wouters, J.-S. Caux, F. H. L. Essler, and T. Prosen, "Complete Generalized Gibbs Ensembles in an Interacting Theory," Phys. Rev. Lett. 115 (2015) no. 15, 157201, arXiv:1507.02993 [quant-ph].

[3] L. Vidmar and M. Rigol, "Generalized Gibbs ensemble in integrable lattice models," J. Stat. Mech. 6 (2016) 064007, arXiv:1604.03990 [cond-mat.stat-mech]

[4] O. A. Castro-Alvaredo, B. Doyon, and T. Yoshimura, "Emergent Hydrodynamics in Integrable Quantum Systems Out of Equilibrium." Phys. Rev. X 6 (2016) no. 4, 041065, arXiv: 1605.07331 [cond-mat.stat-mech]

[5] B. Bertini, M. Collura, J. De Nardis, and M. Fagotti, "Transport in Out-of-Equilibrium XXZ Chains: Exact Profiles of Charges and Currents," Phys. Rev. Lett. 117 (2016) no. 20, 207201, arXiv:1605.09790 [cond-mat.stat-mech].

[6] T. Kinoshita, T. Wenger, and D. S. Weiss, "A quantum Newton's cradle," Nature 440 (2006) 900.

[7] T. Langen, S. Erne, R. Geiger, B. Rauer, T. Schweigler, M. Kuhnert, W. Rohringer, I. E. Mazets, T. Gasenzer, and J. Schmiedmayer, "Experimental observation of a generalized Gibbs ensemble," Science 348 (2015) 207-211, arXiv:1411.7185 [cond-mat.quant-gas]

[8] M. Schemmer, I. Bouchoule, B. Doyon, and J. Dubail, "Generalized HydroDynamics on an Atom Chip," Phys. Rev. Lett. 122 (2019) no. 9, 090601, arXiv:1810.07170 [cond-mat.quant-gas].

[9] N. Malvania, Y. Zhang, Y. Le, J. Dubail, M. Rigol, and D. S. Weiss, "Generalized hydrodynamics in strongly interacting 1D Bose gases," arXiv e-prints (2020), arXiv:2009.06651 [cond-mat.quant-gas].

[10] F. Lange, Z. Lenarčič, and A. Rosch, "Time-dependent generalized Gibbs ensembles in open quantum systems," Phys. Rev. B 97 (2018) no. 16, 165138, arXiv:1801.07646 [cond-mat.str-el]

[11] A. J. Friedman, S. Gopalakrishnan, and R. Vasseur, "Diffusive hydrodynamics from integrability breaking," Phys. Rev. B 101 (2020) no. 18, 180302 . arXiv:1912.08826 [cond-mat.stat-mech].

[12] A. Bastianello, A. De Luca, B. Doyon, and J. De Nardis, "Thermalization of a Trapped One-Dimensional Bose Gas via Diffusion," Phys. Rev. Lett. 125 (2020) 240604, arXiv:2007.04861 [cond-mat.quant-gas].

[13] A. Bastianello, V. Alba, and J.-S. Caux, "Generalized Hydrodynamics with Space-Time Inhomogeneous Interactions," Physical Review Letters 123 (2019) no. 13. arXiv:1906.01654 [cond-mat.stat-mech].

[14] R. Koch, A. Bastianello, and J.-S. Caux, "Adiabatic formation of bound states in the 1d Bose gas," arXiv e-prints (2020), arXiv:2010.13738 [cond-mat.quant-gas]

[15] J. Durnin, M. Bhaseen, and B. Doyon, "Non-Equilibrium Dynamics and Weakly Broken Integrability," arXiv e-prints (2020), arXiv:2004.11030 [cond-mat.stat-mech].

[16] A. Bastianello, J. De Nardis, and A. De Luca, "Generalized hydrodynamics with dephasing noise," Physical Review B 102 (2020) no. 16, arXiv:2003.01702 [cond-mat.stat-mech]. 
[17] I. Bouchoule, B. Doyon, and J. Dubail, "The effect of atom losses on the distribution of rapidities in the one-dimensional Bose gas," SciPost Physics 9 (2020) o. 4, 044, arXiv: 2006.03583 [cond-mat.quant-gas].

[18] G. Lindblad "On the generators of quantum dynamical semigroups," Commun.Math. Phys. 48 (1976) 119-130.

[19] D. Manzano, "A short introduction to the Lindblad haster equation," AIP Advances 10 (2020) no. 2, 025106, arXiv:1906.04478 [quant-ph]

[20] A. Rivas, A. D. K. Plato, S. F. Huelga, and M. B. Flenio, "Markovian master equations: a critical study," New Journal of Physics 12 (2010) no. 11, 113032.

[21] D. Rossini, A. Ghermaoui, M. Bosch Aguilera, R. Vatré, H. Bouganne, J. Beugnon, F. Gerbier, and L. Mazza, "Strong correlations in lossy one-dimensional quantum gases: from the quantum Zeno effect to the generalized Gibbs ensemble," arXiv e-prints (2020) ArXiv:2011.04318 [cond-mat.quant-gas]

[22] L. Sá, P. Ribeiro, and T. Prosen, "Integrable Non-unitary Open Quantum Circuits," arXiv e-prints (2020), arXiv:2011.06565 [cond-mat.stat-mech].

[23] F. Reiter, D. Reeb, and A. S. Sørensen, "Scalable Dissipative Preparation of Many-Body Entanglement," Phys. Rev. Lett. 117 (2016) no. 4, 040501. arXiv:1501.06611 [quant-ph]

[24] Y. Lin, J. P. Gaebler, F. Reiter, T. R. Tan, R. Bowler, . S. Sørensen, D. Leibfried, and D. J. Wineland, "Dissipative production of a maximally entangled steady state of two quantum bits." Nature 504 (2013) no. 7480, 415-418, arXiv: 1307.4443 [quant-ph].

[25] F. Reiter, F. Lange, S. Jain, M. Grau, J. P. Home, and Z. Lenarčič, "Engineering generalized Gibbs ensembles with trapped ions." arXiv e-prints (2019) ArXiv:1910.01593 [quant-ph]

[26] A. Nava, M. Rossi, and D. Giuliano, "Lindblad equation approach to the optimal working point in non equilibrium stationary states of an interacting electronic one-dimensional system: application to the spinless Hubbard chain in the clean and in the weakly disordered limit." arXiv e-prints (2020), arXiv:2010.04533 cond-mat.str-el].

[27] T. Prosen, "Third quantization: a general method to solve master equations for quadratic open Fermi s vstems," New Journal of Physics 10 (2008) no. 4, 43026, arXiv:0801.1257 [quant-ph].

[28] T. Prosen and B. Zunkovič, "Exact solution of Markovian master equations for quadratic Fermi systems: thermal baths, open XY spin chains and non-equilibrium phase transition," New Journal of Physics 12 (2010) o. 2, 025016, arXiv:0910.0195 [quant-ph].

[29] N. Shibata and H. Katsura, "Dissipative spin chain as a non-Hermitian Kitaev ladder," Phys. Rev. B. 99 (2019)| no. 17.174303, arXiv:1812.10373 [cond-mat.stat-mech].

[30] E. Vernier, "Mixing times and cutoffs in open quadratic fermionic systems," SciPost Physics 9 (2020) no. 4, 049, arXiv:2004.11788 [cond-mat.stat-mech].

[31] T. Prosen, "Open XXZ Spin Chain: Nonequilibrium Steady State and a Strict Bound on Ballistic Transport," Phys. Rev. Lett. 106 (2011) no. 21, 217206. arXiv:1103.1350 [cond-mat.str-el]

[32] T. Prosen, "Exact Nonequilibrium Steady State of a Strongly Driven Open XXZ Chain." Phys. Rev. Lett. 107 (2011) no. 13, 137201, arXiv:1106.2978 [quant-ph].
[33] D. Karevski, V. Popkov, and G. M. Schütz, "Exact Matrix Product Solution for the Boundary-Driven Lindblad XXZ Chain." Phys. Rev. Lett. 110 (2013) no. 4, 047201, arXiv:1211.7010 [cond-mat.stat-mech].

[34] T. Prosen, E. Ilievski, and V. Popkov, "Exterior integrability: Yang-Baxter form of non-equilibrium steady-state density operator," New Journal of Physics 15 (2013) no. 7, 073051, arXiv:1304.7944 [math-ph].

[35] E. Ilievski, "Exact solutions of open integrable quantum spin chains," arXiv e-prints (2014), arXiv:1410.1446 [quant-ph].

[36 $[$ V. Popkov and G. M. Schütz, "Solution of the Lindblad equation for spin helix states," Phys. Rev. E 95 (2017) no. 4, 042128, arXiv:1702.04586 [cond-mat.str-el].

[37] B. Buča, C. Booker, M. Medenjak, and D. Jaksch, "Bethe ansatz approach for dissipation: exact solutions of quantum many-body dynamics under loss," New Journa of Physics 22 (2020) no. 12, 123040, arXiv:2004.05955 [cond-mat.stat-mech]

[38] M. Nakagawa, N. Kawakami, and M. Ueda, "Exact Liouvillian Spectrum of a One-Dimensional Dissipative Hubbard Model," arXiv e-prints (2020) arXiv:2003.14202 [cond-mat.quant-gas].

[39] F. H. L. Essler and L. Piroli, "Integrability of $1 D$ Lindbladians from operator-space fragmentation," Phys. Rev. E 102 (2020) 062210, 2009.11745 [cond-mat.stat-mech].

[40] M. V. Medvedyeva, F. H. L. Essler, and T. Prosen, "Exact Bethe Ansatz Spectrum of a Tight-Binding Chain with Dephasing Noise," Phys. Rev. Lett. 117 (2016) no. 13, 137202, arXiv:1606.09122 [quant-ph].

[41] N. Shibata and H. Katsura, "Dissipative quantum Ising chain as a non-Hermitian Ashkin-Teller model," Phys. Rev. B. 99 (2019) no. 22, 224432, arXiv:1904.12505 [cond-mat.stat-mech]

[42] A. A. Ziolkowska and F. H. L. Essler, "Yang-Baxter integrable Lindblad equations," SciPost Phys. 8 (2019) 044, arXiv:1911.04883 [cond-mat.stat-mech].

[43] V. Korepin, N. Bogoliubov, and A. Izergin, Quantum inverse scattering method and correlation functions. Cambridge University Press, 1993.

[44] M. De Leeuw, A. Pribytok, and P. Ryan, "Classifying two-dimensional integrable spin chains," J. Phys. A 52 (2019) no. 50, 505201, arXiv:1904.12005 [math-ph].

45] M. de Leeuw, C. Paletta, A. Pribytok, A. L. Retore, and P. Ryan, "Classifying Nearest-Neighbor Interactions and Deformations of AdS," Phys. Rev. Lett. 125 (2020) no. 3, 031604, arXiv:2003.04332 [hep-th].

446 M. De Leeuw, A. Pribytok, A. L. Retore, and P. Ryan, "New integrable 1D models of superconductivity," J. Phys. A 53 (2020) no. 38, 385201, arXiv:1911.01439 [math-ph]

[47] M. de Leeuw, C. Paletta, A. Pribytok, A. L. Retore, and P. Ryan, "Yang-Baxter and the Boost: splitting the difference," arXiv e-prints (2020), arXiv:2010.11231 [math-ph].

[48] D. Manzano and P. Hurtado, "Harnessing symmetry to control quantum transport," Advances in Physics $\mathbf{6 7}$ (2018) no. 1, 1-67.

[49] H.-P. Breuer, F. Petruccione, et al., The theory of open quantum systems. Oxford University Press on Demand, 2002.

[50] G. Schütz, Integrable Stochastic Many-body Systems. Berichte des Forschungszentrums Jülich. 
Forschungszentrum, Zentralbibliothek, 1998.

[51] K. Temme, M. M. Wolf, and F. Verstraete, "Stochastic exclusion processes versus coherent transport," Nev Journal of Physics 14 (2012) no. 7, 075004 arXiv:0912.0858 [quant-ph].

[52] V. Eisler, "Crossover between ballistic and diffusive transport: the quantum exclusion process," J. Stat. Mech. 2011 (2011) no. 6, 06007, arXiv:1104.4050 [cond-mat.stat-mech].

[53] S. Sandow, "Partially asymmetric exclusion process with open boundaries," Physical Review E 50 (1994) no. 4. 2660-2667, arXiv: cond-mat/9405073 [cond-mat].

[54] M. Tetelman, " Lorentz group for two-dimensional integrable lattice svstems." Sov. Phys. JETP 55(2) (1982) 306-310.

[55] J. Links, H.-Q. Zhou, R. H. McKenzie, and M. D. Gould, "Ladder Operator for the One-Dimensional Hubbard Model," Phys. Rev. Lett. 86 (2001) no. 22. 5096-5099, arXiv: cond-mat/0011368 [cond-mat.str-el].

[56] F. Loebbert, "Lectures on Yangian Symmetry," J. Phys. 49 (2016) no. 32, 323002, arXiv:1606.02947 hep-th].

[57] F. C. Alcaraz and W. F. Wreszinski, "The Heisenberg XXZ Hamiltonian with Dzyaloshinsky-Moriya interactions," Journal of Statistical Physics 58 (1990) no. 1, 45-56. https://doi .org/10.1007/BF01020284.

[58] We omit some models with less interesting physical properties, such as those with diagonal $h$ and $\ell$ operators. A complete list will be presented elsewhere.

[59] Supplemental Materials to "Constructing Integrable Lindblad Superoperators".

[60] A. Lushnikov, "Binary reaction $1+1 \rightarrow 0$ in one dimension," Physics Letters A 120 (1987) no. 3, 135 137 .

[61] A. Lushnikov, "Binary reaction $1+1 \rightarrow 0$ in one dimension," Sov. Phys. JETP 64 (1986) no. 4, 1376.

[62] J. E. Santos, G. M. Schütz, and R. B. Stinchcombe, "Diffusion-annihilation dynamics in one spatial dimension," The Journal of Chemical Physics 105 (1996) no. 6, 2399-2407.

[63] F. H. L. Essler, H. Frahm, F. Göhmann, A. Klümper, and V. E. Korepin, The One-Dimensional Hubbard Mode. Cambridge University Press, Cambridge, 2005.

[64] S. Murakami, "New integrable extension of the Hubbard chain with variable range hopping," Journal of Physics A: Mathematical and General 31 (1998) no. 30, 6367-6384, arXiv: cond-mat/9802066 [cond-mat.stat-mech].

65] F. Lange, Z. Lenarčič, and A. Rosch, "Pumping approximately integrable systems." Nature Communications 8 (2017) 15767, arXiv:1608.03563 [cond-mat.str-el]].

[66] K. Yamamoto, Y. Ashida, and N. Kawakami, "Rectification in nonequilibrium steady states of open many-body systems," Physical Review Research 2 (2020) no. 4, 043343, arXiv:2009.00838 


\section{Supplemental Material:}

\section{Constructing Integrable Lindblad Superoperators}

\section{NOTATIONS}

Below we present a list of the integrable Lindbladians treated in the main text. For each model we give here the matrix representation of the Hamiltonian density $h$ and the jump operator $\ell$. We also present the corresponding $R$-matrix, solution of the Yang-Baxter equation

$$
R_{12}\left(u_{1}, u_{2}\right) R_{13}\left(u_{1}, u_{3}\right) R_{23}\left(u_{2}, u_{3}\right)=R_{23}\left(u_{2}, u_{3}\right) R_{13}\left(u_{1}, u_{3}\right) R_{12}\left(u_{1}, u_{2}\right) .
$$

We apply the following conventions. The components of a single spin are denoted as

$$
\left(\begin{array}{c}
\psi_{\uparrow} \\
\psi_{\downarrow}
\end{array}\right)
$$

For the tensor product of two spins we apply the notation

$$
\left(\begin{array}{c}
\psi_{\uparrow} \\
\psi_{\downarrow}
\end{array}\right) \otimes\left(\begin{array}{c}
\psi_{\uparrow} \\
\psi_{\downarrow}
\end{array}\right) \equiv\left(\begin{array}{c}
\psi_{\uparrow \uparrow} \\
\psi_{\uparrow \downarrow} \\
\psi_{\downarrow \uparrow} \\
\psi_{\downarrow \downarrow}
\end{array}\right) .
$$

The density matrix of a two-site segment is given by

$$
\rho_{a b}^{c d}=\psi_{a b} \psi_{c d}^{*}, \quad a, b, c, d=\uparrow, \downarrow .
$$

In the superoperator formalism we treat such a density matrix as a vector with 16 components. The identification of the components is given by

$$
\rho=\left(\begin{array}{cccc}
\rho_{\uparrow \uparrow}^{\uparrow \uparrow} & \rho_{\uparrow \uparrow}^{\uparrow \downarrow} & \rho_{\uparrow \uparrow}^{\downarrow \uparrow} & \rho_{\uparrow \uparrow}^{\downarrow \downarrow} \\
\rho_{\uparrow \uparrow}^{\uparrow \uparrow} & \rho_{\uparrow \downarrow}^{\uparrow \downarrow} & \rho_{\uparrow \uparrow}^{\downarrow \uparrow} & \rho_{\uparrow \downarrow}^{\downarrow \downarrow} \\
\rho_{\downarrow \uparrow}^{\uparrow \uparrow} & \rho_{\downarrow \uparrow}^{\uparrow \downarrow} & \rho_{\downarrow \uparrow}^{\downarrow \uparrow} & \rho_{\downarrow \uparrow}^{\downarrow \downarrow} \\
\rho_{\downarrow \uparrow} & \rho_{\downarrow \downarrow}^{\uparrow \downarrow} & \rho_{\downarrow \uparrow}^{\downarrow \uparrow} & \rho_{\downarrow \downarrow}^{\downarrow \downarrow}
\end{array}\right)=\left(\begin{array}{cccc}
\rho_{1} & \rho_{2} & \rho_{3} & \rho_{4} \\
\rho_{5} & \rho_{6} & \rho_{7} & \rho_{8} \\
\rho_{9} & \rho_{10} & \rho_{11} & \rho_{12} \\
\rho_{13} & \rho_{14} & \rho_{15} & \rho_{16}
\end{array}\right) .
$$

The $4 \times 4$ matrices $h(u)$ and $\ell(u)$ act on the vectors of the two-site segment as given by (53). The superoperator $\mathcal{L}(u)$ can be represented by a matrix of size $16 \times 16$ and it acts on the components of $\rho$ as indexed by (S5).

For the $R$-matrix we will denote by $R_{i}^{j}$ the element corresponding to $j$-row and $i$-column. The $R$-matrix satisfies the conditions

$$
R(u, u)=P, \quad \mathcal{L}(u)=\left.P \partial_{v} R(v, u)\right|_{v=u},
$$

with $P$ being the permutation operator acting on two copies of $\mathbb{C}^{4}$, and

$$
\mathcal{L}(u)=-i h^{(1)}(u)+i h^{(2) *}(u)+\ell^{(1)}(u) \ell^{(2) *}(u)-\frac{1}{2} \ell^{(1) \dagger}(u) \ell^{(1)}(u)-\frac{1}{2} \ell^{(2) T}(u) \ell^{(2) *}(u) .
$$

Here ${ }^{T}$ denotes transpose and the asterisk stands for complex conjugation element wise.

If the $R$-matrix is of difference form, that is $R(v, u)=R(v-u)$, then the superoperator does not depend on $u$ and we will suppress it in the notation.

We choose the multiplicative normalization of the $R$-matrix such that (S6) are satisfied.

In all the models, $\phi$ is a real constant. 


\section{MODEL A1}

We have

$$
h=\frac{1}{2}\left(\begin{array}{cccc}
0 & 0 & 0 & 0 \\
0 & 0 & e^{i \phi} & 0 \\
0 & e^{-i \phi} & 0 & 0 \\
0 & 0 & 0 & 0
\end{array}\right), \quad \ell=\left(\begin{array}{cccc}
0 & 0 & 0 & 0 \\
0 & 0 & 0 & 0 \\
0 & 1 & -i e^{i \phi} & 0 \\
0 & 0 & 0 & 0
\end{array}\right)
$$

The non-zero entries of the $R$-matrix are

$$
\begin{aligned}
& R_{1}^{1}=R_{13}^{4}=R_{6}^{6}=R_{11}^{11}=R_{16}^{16}=1 \\
& R_{5}^{2}=R_{2}^{5}=R_{9}^{3}=R_{3}^{9}=R_{14}^{8}=R_{8}^{14}=R_{15}^{12}=R_{12}^{15}=e^{-\frac{u}{2}} \\
& -\frac{i R_{2}^{2}}{e^{i \phi}}=i e^{i \phi} R_{3}^{3}=R_{4}^{4}=i e^{i \phi} R_{8}^{8}=-\frac{i R_{12}^{12}}{e^{i \phi}}=2 e^{-\frac{u}{2}} \sinh \left(\frac{u}{2}\right) \\
& -e^{i \phi} R_{4}^{7}=\frac{R_{4}^{10}}{e^{i \phi}}=2 i e^{-u} \sinh \left(\frac{u}{2}\right) \\
& R_{10}^{7}=R_{7}^{10}=R_{4}^{13}=e^{-u}
\end{aligned}
$$

An alternative representation for the same super-operator can be obtained by an additive shift to $\ell$, leading to

$$
h=0, \quad \ell=i e^{i \phi}\left(\begin{array}{cccc}
1 & 0 & 0 & 0 \\
0 & 1 & 0 & 0 \\
0 & -i e^{-i \phi} & 0 & 0 \\
0 & 0 & 0 & 1
\end{array}\right) .
$$

\section{MODEL A2}

We have

$$
h=\frac{\tau}{2}\left(\begin{array}{cccc}
0 & 0 & 0 & 0 \\
0 & 0 & i & 0 \\
0 & -i & 0 & 0 \\
0 & 0 & 0 & 0
\end{array}\right), \quad \ell=\left(\begin{array}{cccc}
1 & 0 & 0 & 0 \\
0 & 0 & 0 & 0 \\
0 & \tau & 1 & 0 \\
1 & 0 & 0 & 0
\end{array}\right)
$$

with $\tau= \pm 1$.

The non-zero entries of the $R$-matrix are

$$
\begin{aligned}
& R_{16}^{16}=R_{13}^{4}=1 \\
& \tau R_{3}^{8}=\tau R_{2}^{12}=-\tau R_{4}^{7}=-\tau R_{4}^{10}=R_{1}^{6}=R_{1}^{11}=2 e^{-u} \sinh \left(\frac{u}{2}\right), \\
& -\tau R_{12}^{12}=-\tau R_{8}^{8}=R_{4}^{4}=R_{9}^{8}=R_{5}^{12}=R_{1}^{16}=1-e^{-u} \\
& R_{5}^{2}=R_{9}^{3}=R_{14}^{8}=R_{8}^{14}=R_{15}^{12}=R_{12}^{15}=e^{-\frac{u}{2}} \\
& R_{3}^{9}=R_{2}^{5}=e^{-\frac{3 u}{2}} \\
& R_{1}^{1}=R_{6}^{6}=R_{11}^{11}=R_{10}^{7}=R_{7}^{10}=R_{4}^{13}=e^{-u} .
\end{aligned}
$$

\section{MODEL B1}

We have

$$
h=0, \quad \ell=\left(\begin{array}{cccc}
\tau & 0 & 0 & 0 \\
0 & 0 & 1 & 0 \\
0 & 1 & 0 & 0 \\
0 & 0 & 0 & \kappa
\end{array}\right),
$$


$\tau= \pm 1$ and $\kappa= \pm 1$.

The non-zero entries of the $R$-matrix are

$$
\begin{aligned}
& R_{1}^{1}=R_{16}^{16}=e^{-u}(u+1), \\
& R_{6}^{6}=R_{11}^{11}=e^{-u}(\kappa \tau u+1), \\
& R_{5}^{2}=R_{2}^{5}=R_{9}^{3}=R_{3}^{9}=R_{13}^{4}=R_{4}^{13}=R_{10}^{7}=R_{7}^{10}=R_{14}^{8}=R_{8}^{14}=R_{15}^{12}=R_{12}^{15}=e^{-u}, \\
& \tau R_{2}^{2}=\tau R_{3}^{3}=\tau R_{5}^{5}=\tau R_{9}^{9}=\kappa R_{8}^{8}=\kappa R_{12}^{12}=\kappa R_{14}^{14}=\kappa R_{15}^{15}=R_{4}^{4}=R_{7}^{7}=R_{10}^{10}=R_{13}^{13}=e^{-u} u .
\end{aligned}
$$

Remarkably, for $\tau=\kappa=1$

$$
R(u)=\frac{u}{e^{u}}\left(1+\frac{1}{u} P\right)
$$

which is the $R$-matrix of the $S U(4)$ invariant chain (apart from an irrelevant pre-factor).

\section{MODEL B2}

We have

$$
h=\frac{1}{2}\left(\begin{array}{cccc}
0 & 0 & 0 & 0 \\
0 & 0 & e^{i \phi} & 0 \\
0 & e^{-i \phi} & 0 & 0 \\
0 & 0 & 0 & 0
\end{array}\right), \quad \frac{\ell(u)}{\beta(u)}=\left(\begin{array}{cccc}
\cosh (u) & 0 & 0 & 0 \\
0 & 1 & i e^{i \phi} \sinh (u) & 0 \\
0 & -i \frac{\sinh (u)}{e^{i \phi}} & -1 & 0 \\
0 & 0 & 0 & -\cosh (u)
\end{array}\right) \text {, }
$$

and

$$
\beta(u)=\sqrt[4]{\frac{\gamma}{2 \gamma \cosh (2 u)+2}}
$$

with $\gamma \geq 0$ being a fixed coupling constant.

This model is equivalent to the Hubbard model, and it was already studied in [51], see Section 4.3.1 in that work. Our formulas involve a simple reparametrization of the coupling, and the additional twist $e^{i \phi}$. These changes are easily accomodated into the known $R$-matrix, displayed for example in [52].

\section{MODEL B3}

We have

$$
h=\frac{1}{2}\left(\begin{array}{cccc}
0 & 0 & 0 & 0 \\
0 & 0 & e^{i \phi} & 0 \\
0 & e^{-i \phi} & 0 & 0 \\
0 & 0 & 0 & 0
\end{array}\right), \quad \ell=\sqrt{\frac{\gamma}{2}}\left(\begin{array}{cccc}
\gamma & 0 & 0 & 0 \\
0 & 1 & i(\gamma-1) e^{i \phi} & 0 \\
0 & -i(\gamma+1) e^{-i \phi} & -1 & 0 \\
0 & 0 & 0 & \gamma
\end{array}\right)
$$

with $\gamma \geq 0$ being a coupling constrant. 
The non-zero entries of the $R$-matrix are

$$
\begin{aligned}
& R_{1}^{1}=R_{6}^{6}=R_{11}^{11}=R_{16}^{16}=1, \\
& \frac{R_{2}^{2}}{e^{i \phi}}=-e^{i \phi} R_{3}^{3}=-e^{i \phi} R_{8}^{8}=\frac{R_{12}^{12}}{e^{i \phi}}=\frac{i(\gamma+1)}{\gamma+\operatorname{coth}(\alpha(u))}, \\
& -e^{i \phi} R_{5}^{5}=\frac{R_{9}^{9}}{e^{i \phi}}=\frac{R_{14}^{14}}{e^{i \phi}}=-e^{i \phi} R_{15}^{15}=\frac{i(\gamma-1)}{\gamma+\operatorname{coth}(\alpha(u))}, \\
& R_{5}^{2}=R_{2}^{5}=R_{12}^{15}=R_{15}^{12}=R_{14}^{8}=R_{8}^{14}=R_{3}^{9}=R_{9}^{3}=\frac{1}{\gamma \sinh (\alpha(u))+\cosh (\alpha(u))}, \\
& e^{i \phi} R_{7}^{4}=-\frac{R_{10}^{4}}{e^{i \phi}}=\frac{R_{10}^{13}}{e^{i \phi}}=-e^{i \phi} R_{7}^{13}=\frac{2 i\left(\gamma^{2}-1\right) \sinh (\alpha(u))}{\zeta(u)}, \\
& -e^{i \phi} R_{4}^{7}=R_{4}^{10}=\frac{2 i(\gamma+1)^{2} e^{i \phi} \sinh (\alpha(u))}{\zeta(u)} \\
& e^{i \phi} R_{13}^{7}=-\frac{R_{13}^{10}}{e^{i \phi}}=\frac{2 i(\gamma-1)^{2} \sinh (\alpha(u))}{\zeta(u)}, \\
& R_{7}^{10}=R_{10}^{7}=\frac{2\left(\gamma^{2}+1\right)}{\zeta(u)} \\
& e^{2 i \phi} R_{7}^{7}=\frac{R_{10}^{10}}{e^{2 i \phi}}=-\frac{2\left(\gamma^{2}-1\right) \sinh (\alpha(u))(\gamma \cosh (\alpha(u))+\sinh (\alpha(u)))}{\zeta(u)}, \\
& R_{4}^{4}=\frac{2(\gamma+1)^{2} \sinh (\alpha(u))(\gamma \cosh (\alpha(u))+\sinh (\alpha(u)))}{\zeta(u)}, \\
& R_{13}^{4}=\frac{4 e^{2 \alpha(u)}\left(-\gamma+(\gamma+1) \gamma e^{2 \alpha(u)}+1\right)}{-(\gamma-1)^{3}-2\left(\gamma^{2}-1\right) e^{2 \alpha(u)}+(\gamma+1)^{3} e^{4 \alpha(u)}}, \\
& R_{4}^{13}=-\frac{4\left((\gamma-1) \gamma+(\gamma+1) e^{2 \alpha(u)}\right)}{(\gamma-1)^{3}+2\left(\gamma^{2}-1\right) e^{2 \alpha(u)}-(\gamma+1)^{3} e^{4 \alpha(u)}}, \\
& R_{13}^{13}=\frac{2(\gamma-1)^{2} \sinh (\alpha(u))(\gamma \cosh (\alpha(u))+\sinh (\alpha(u)))}{\zeta(u)},
\end{aligned}
$$

where $\alpha(u)=\frac{1}{2}\left(\gamma^{2}+1\right) u$ and $\zeta(u)=1-\gamma^{2}+\gamma^{3} \sinh (2 \alpha(u))+3 \gamma^{2} \cosh (2 \alpha(u))+3 \gamma \sinh (2 \alpha(u))+\cosh (2 \alpha(u))$.

[S1] A. A. Ziolkowska and F. H. L. Essler, "Yang-Baxter integrable Lindblad equations," SciPost Phys. 8 (2019) 044, arXiv: 1911.04883 [cond-mat.stat-mech].

[S2] F. H. L. Essler, H. Frahm, F. Göhmann, A. Klümper, and V. E. Korepin, The One-Dimensional Hubbard Mode Cambridge University Press, Cambridge, 2005. 